Dies- und Jenseits des Endes der Geschichte, Helena

Author(s): Fritz Breithaupt

Source: MLN, Vol. 114, No. 3, German Issue (Apr., 1999), pp. 528-550

Published by: The Johns Hopkins University Press

Stable URL: http://www.jstor.org/stable/3251446

Accessed: 13-06-2017 16:11 UTC

JSTOR is a not-for-profit service that helps scholars, researchers, and students discover, use, and build upon a wide range of content in a trusted digital archive. We use information technology and tools to increase productivity and facilitate new forms of scholarship. For more information about JSTOR, please contact support@jstor.org.

Your use of the JSTOR archive indicates your acceptance of the Terms \& Conditions of Use, available at

http://about.jstor.org/terms

The Johns Hopkins University Press is collaborating with JSTOR to digitize, preserve and extend access to $M L N$ 


\title{
Dies- und Jenseits des Endes der Geschichte, Helena
}

\author{
ช \\ Fritz Breithaupt
}

In den Diskussionen um die Bestimmung der Epochen einerseits und ihrer Schwellen andrerseits, der Paradigmen und ihrer Wechsel, ist sonderbarerweise eine Verbindung selten hergestellt worden: daß nämlich die Epoche ihren eigenen Bruch heraufbeschwört. Wer den Begriff der Epoche für legitimiert hält, wird sich dazu genötigt sehen anzunehmen, daß eine Epoche auf einem ihr eigenen Prinzip (oder mehreren) beruht-sei's ein Wesen, Paradigma, Affekt, eine Codierung oder Leitdifferenz-durch welches sie sich als Epoche konsolidiert. Zwei prinzipielle Szenarien des Epochenbruchs sind denkbar: zum einen der Verlust dieses Leitprinzips, vielleicht durch von außen kommende Störung, zum anderen nicht die Abschwächung, sondern eben die Verstärkung des Leitprinzips, seine Überfunktion, dergestalt daß die Epoche an ihrer eigenen Tendenz zugrunde geht. Eben diese zweite Möglichkeit ist die These des alten Goethe, deren Zuspitzung heißt, daß das Wesen einer Epoche nur in der "Schönheit" bestehen kann. Geschichte, epochale Geschichte, kann, so Goethes These, nur diejenige der Schönheit sein. Das heißt nicht, daß nur den Werken der Künstler Geschichtsmächtigkeit zukommt, sondern vielmehr, daß alle gesellschaftlichen und kulturellen Formationen einer Epoche sich in einer Gestaltung der Schönheit verdichten und daß der eigentlich geschichtliche Akt das Wesen der Schönheit sowohl betrifft als auch aus ihm resultiert.

Jede Epoche hat die ihr eigene Schönheit. Schönheit ist das formale Erscheinungsgesetz, welches es den Phänomenen erlaubt, 
sich zu manifestieren, ist ihre Phänomenalität. ${ }^{1}$ Als solches ist sie dasjenige, was dem Prozeß des Erscheinens notwendig entgleitet, nämlich seine Bedingung oder Voraussetzung. Indem sich aber eine Epoche ihrer selbst vergewissert, zur Legitimierung ihrer Selbsterhaltung, das heißt, im Versuch der Abwehr dessen, was in ihr nicht zu erscheinen und marginal zu verbleiben hat, wird eben das Erscheinen der Schönheit gefordert. Doch da das Erscheinen selbst nicht erscheinen kann, ist das Wesen dieses Erscheinens des Erscheinens, die "Gestalt aller Gestalten," ebenso instabil, wie die von ihr dependierenden Phänomene einer Epoche. ${ }^{2}$ Anders gesagt, das Erscheinen kann seinerseits nur erscheinen, kann sich dabei nicht dauerhaft konsolidieren als eine Entität. Indem dieses innerste Wesen der Epoche zur Darstellung gezwungen wird, bricht es auseinander. Der Wille zur Epoche, auch der Wille ihrer Darstellung, fordert ihren Zerfall. Die Schönheit, gerade indem sie zur Geltung kommt, zerbricht am eigenen Exzeß. Suchte Faust zu Beginn nach dem, "was die Welt im Innersten zusammenhält" (382-83), so zeigt Faust II, daß eben dieses Innerste einer Welt als ihr Abgründigstes selbst keinen Halt hat und die Welt dem Zusammensturz aussetzt.

Goethe denkt die Phänomenalität, die Schönheit, nicht nur als eine eigenständige Macht, sondern zudem als eine sich von den Phänomenen ablösende Instanz. In jedem Zur-Erscheinung-Kommen spaltet sich von dem Phänomen seine eigene Phänomenalität ab. Diese Phänomenalität, das "Musterbild," die "Gestalt aller Gestalten," die eben nicht nur eine ausgezeichnete Gestalt unter anderen, sondern das Prinzip des Morphischen selbst ist, die "Schöngestalt" (8532), personifiziert Goethe in Helena, der Helena, die in Faust II durch die Zeit wandert. In ihr kristallisiert Goethe, was eine Epoche ausmacht, was ihr gesamtes Sinngefüge organisiert, selbst aber nicht rational gegründet ist. Helena ist die "Laterna magica," in deren

\footnotetext{
${ }^{1} \mathrm{Daß}$ Schönheit in der Phänomenalität besteht, ist eine der Grundthesen Goethes, zu der er bereits in den frühen Versuchen zur Optik ab 1792 gelangt und die er auch in den ästhetischen und morphologischen Texten nach der Italienischen Reise verfolgt, etwa in Inwiefern die Idee: Schönheit sei Vollkommenheit mit Freiheit, auf organische Naturen angewendet werden könne (1794). Statt einer umfangreichen Herleitung dieses Axioms sollen die folgenden Ausführungen seine Konsequenzen beleuchten. Einige in diesem Kontext relevante Thesen zur Phänomenalität entwickelt Christiaan L. Hart Nibbrig, Spiegelschrift, Frankfurt 1987, 193-209.

${ }^{2}$ Aus Goethes Faust wird im Folgenden nur unter Angabe der Verszahl nach der Frankfurter Ausgabe (FA) zitiert, Johann Wolfgang von Goethe, Sämtliche Werke. Briefe, Tagebücher und Gespräche, Band I/7, hrsg. von Albrecht Schöne, Frankfurt 1994, hier: 6185.
} 
Projektionen die Geister einer Epoche zum Leben kommen, solange man nicht unmittelbar in ihr Licht schaut (6377-565). Helena droht stets, an sich selbst zu zerbrechen.

"Die Schönheit kann nie über sich selbst deutlich werden," lautet ein Satz der Maximen und Reflexionen (Nr. 726). ${ }^{3}$ So wird Helena einmal zu der Einsicht genötigt, daß sie selbst nicht mehr als ein Idol ist, um daraufhin beinahe zu entschwinden. Sie sagt, auf den toten Achilles bezogen: "Ich als Idol, Ihm dem Idol verband ich mich. Es war ein Traum, so sagen ja die Worte selbst. Ich schwinde hin und werde selbst mir ein Idol." Danach die Bühnenanweisung: "Sinkt dem Halbchor in die Arme" (8879-81). Wer sich selbst zum Idol, zum Bild oder Traum wird, hört auf, er selbst zu sein (ein Paralipomenon spricht von ihrem "NichtigkeitsGefühl"). Er hört nicht einmal auf, er selbst zu sein, da eben dieses Selbst sich als ein Traum erweist. Ohne ein solches Selbst aber, und dies ist der eigentliche Skandal der Aussage Helenas, hört sie sogar auf, ein Bild oder Idol zu sein, da ein Bild stets Bild von etwas ist. Die Struktur des "von etwas" ist die Struktur alles Bildlichen. Wenn nun aber Helena, die an dieser Stelle sogleich als "Gestalt aller Gestalten" betitelt wird, sowohl aufhört, sie selbst, als auch überhaupt zu sein, dann kann sie nicht mehr als der Prototyp aller Gestalten fungieren. Sie kann nur noch der Prototyp einer entleerten Bildlichkeit sein, die Bilder bestenfalls von Bildern bietet, ohne aber Bild von etwas zu sein. Ohne sie wäre ein jedes Phänomen nichts als ein bloßes Phantom: und tatsächlich fallen die Wesen nach Helenas Entfernen den Gespenstern anheim. Ohne Helenas Licht zerfällt die überzeitliche Stabilität; es "verdüstert allzugleich/ Mit dem Glanz der Gegenwart/ Auch der Zukunft/ Mild aufschimmerndes Hoffnungslicht” (8889-902). Helena, der Prototyp alles Bildlichen, darf und kann selbst nicht nur ein bloßes Bild sein, denn damit ware alles Bildliche ohne Licht und Orientierung, da ungewiß wäre, wovon es Bild ist und ob es überhaupt noch ein Bildliches sein kann; zugleich aber muß Helena ein Bild sein, ein Phänomen, das die anderen Phänomene in ihrer Erscheinung leitet. In ihr berühren sich zwei konträre Tendenzen dergestalt, daß Helena weder als ein Wesen zu denken ist, noch daß sie als bloß unzugängliche Idee gelten kann. Sie muß, ohne es zu können, beides sein. Ihre aporetische Konstitution findet ihren Ausdruck-den einzigen an-

${ }^{3}$ Zur Diskussion dieser Wendung vergleiche Walter Benjamin, Goethes Wahlverwandtschaften, in: Gesammelte Schriften, hrsg. von Rolf Tiedemann und Hermann Schweppenhäuser, Frankfurt 1974, Band I, 197. 
gemessenen Ausdruck, den sie haben kann, da er nichts zeigt und im Ausdruck dem Ausdruckslosen anheimfällt-in ihrer Explosivität; als Faust sie am Ende des Schattenspiels (Faust II, 2 Akt) leibhaft zu greifen sucht, explodiert sie. ${ }^{4}$

Eine jede Kristallisation der Schönheit, des Idols und der "Gestalt aller Gestalten" ist haltlos. $\mathrm{Da} ß$ sie dennoch hält, "verharr[t]" und

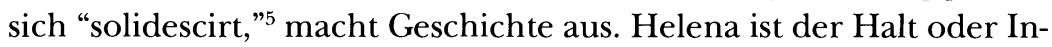
Halt, der die Epoche verankern soll und dessen "halte[n], festhalte[n]" der Chor sogleich beschwört (8906), der jedoch keinen Halt außerhalb von sich selbst finden kann und darf. Der Widerstand des Haltlosen gegen das Schwanken der Zeit, der Aufschub des Untergangs, ist die Essenz von Kultur und Epoche. Vielleicht ist eben dies der Grund, warum Faust Helena aus der Vorwelt der Mütter gewinnen muß. Mütter sind diejenigen, die dem Anspruch der Zeit trotzen, indem sie als Wesen der Generation und Regeneration Zeit aufheben. ${ }^{6}$ Nur an diesem Anspruch auf Bestand und Geltung kann sich Geschichte überhaupt erweisen: als der Zusammenbruch dieses Anspruchs. Nicht Zufall und Vergänglichkeit, sondern der Widerstand gegen Zufall und Vergänglichkeit erlaubt das Denken von Geschichte. Eben dieser Widerstand, das Entgegenstehen gegen den Lauf der Zeit, wird vom Phänomenalen, vom Gegenständlichen, geleistet. Und zwar nicht vom einzelnen Phänomen oder Gegenstand, sondern von der Phänomenalität als dem Gegenstehen des Gegenständlichen, als der Kraft, die es dem einzelnen Phänomen erlaubt, überhaupt zu

\footnotetext{
${ }^{4}$ Die Inkonsistenz von Helena und Euphorion hat Benjamin Bennett zu der These veranlaßt, daß Goethe in ihnen die Struktur von Witz und Ironie vorführe, die die essentielle Leere dieser Geschöpfe der Poesie bloßstelle. Dieser Überlegung wäre hinzuzufügen, daß Geschichte im Faust II in der Tat strukturelle Nähe zum Witz besitzt, da Geschichte als Selbstzerstörung der Epoche auftritt. Dagegen kann ich Bennetts Folgethese, daß die Ironie die Union von Helena und Faust, von dem Klassischen und der Romantik, vorbereite, nicht folgen; Goethe's Theory of Poetry: "Faust" and the Regeneration of Language, Ithaca und London 1986, 136-161.

${ }^{5}$ Als Goethe über seine Schöpfung der Helena reflektiert, hebt er eben dieses Moment ihrer Verdichtung zu einem Wesen hervor, die Gestaltung, die der Umgestaltung trotzt: "wie vielfach sich diese [Helena] in langen, kaum übersehbaren Jahren gestaltet und umgestaltet, nun mag sie im Zeitmoment solidcirt endlich verharren" (Brief an Nees von Esenbeck, 24 Mai 1827, WA IV, 42, 198.) Eben weil sie nun eingefroren ist, sich der Zeit widersetzt, steht sie mit momentum "im Zeitmoment."

${ }^{6}$ Diese These vertritt die materialreiche Studie von Harold Jantz, The Mothers in Faust: The Myth of Time and Creativity, Baltimore 1969. Er referiert auch eine, Goethe höchstwahrscheinlich bekannte, Version des Muttermythos des Diodorus Siculus, daß die Urmutter Rhea mit Hilfe der Korybanten Zeus unbemerkt von dem allesverschlingenden Chronos aufgezogen habe. Die Funktion der Urmutter ist so unmittelbar die Abwehr der Zeit.
} 
erscheinen, namentlich der Schönheit. Solange Helena steht, besteht die Epoche; verabschiedet sich Helena, so fällt mit ihr das Zeitalter.

$\mathrm{Da} \beta$ es Geschichte geben kann, resultiert aus der die Phänomene formierenden Schönheit: doch daß es Geschichte geben soll, verdankt sich dem von der Schönheit Ausgeschlossenen und Nicht-Zugelassenen: dem Marginalen und Nicht-Erscheinenden. Nur so lange etwas nicht erscheint, bleibt etwas, was-in einer anderen Zeit, einer Zeit anders als die Zeit der Epochen vielleicht—erscheinen könnte. Und so ist das Pendant von Helena, die in Phorkyas verkörperte Häßlichkeit, vor allem das Anästhetische: dasjenige, das nicht zur Erscheinung kommt, was der Nacht angehört, die "Nachtgeburten" (8695) und "Schatten" (8743). ${ }^{7}$ So wie Helena nicht "schön" ist, ist Phorkyas nicht schlicht ein "Häßliches," sondern vielmehr das NichtErscheinende, das Kontur- und Gesichtslose, Amorphe, dessen Auftreten nicht anders als unheimlich, grauenhaft und monströs sein kann, da es nicht gebannt, nicht erkannt, nicht eingegrenzt werden kann. Daher existiert Phorkyas für den Gott der Schönheit, Sonnengott Phöbus, auch überhaupt nicht: "Denn das Häßliche schaut Er nicht, wie sein heilig Auge noch nie erblickte den Schatten" (8741-43). ${ }^{8}$ In der Wahrnehmung eines Phöbus kann es kein Häßliches geben, weil sein sonnengleiches Auge selbst das Licht wirft, welches die Erscheinungen hervortreten läßt und die nicht erscheinenden Schatten, das Häßliche, aufhebt. Apoll ist damit der Geschichtsunterdrücker und Ordnungsbewahrer, vor dem das Anästhetische keine Geltung hat. Falls Phorkyas ein Phänomen ist, dann höchstens dasjenige der Nicht-Phänomenalität. Sie ist das gespensterhafte Gegenstück zur prosopopeia, da sie in der Verkörperung des Anästhetischen nur dessen Entkörperung und in der Gesichtsvergabe als Einäugige und Einzahnige nur den Entzug des Gesichts vorführt (8733).

In Phorkyas pocht im Nicht-Erscheinenden das Marginale auf Geltung, das Vergessene, die Verlierer, diejenigen, die, wörtlich, ein Schattendasein führen, die "an der äußersten Grenze der Erde" hausen. ${ }^{9}$ Und so erscheint sie stets am "Rand," auf der "Schwelle," an der "Grenze," im "Proszenium," verweist als Wärterin des Marginalen

\footnotetext{
${ }^{7}$ Helena bemerkt mit Erstaunen, daß Phorkyas hier tatsächlich ins Sonnenlicht, ins Reich des Erscheinenden tritt: "sie wagt sich sogar ans Licht hervor [...]. Die grausen Nachtgeburten drängt der Schönheitsfreund Phöbus hinweg in die Höhlen, oder bändigt sie" (8693-97).

${ }^{8}$ Vergleiche auch die Schmährede des Chors: "Wagest Du Scheusal neben der Schönheit Dich vor dem Kennerblick Phöbus' zu zeigen?” (8736-39).

${ }^{9}$ Karl Philipp Moritz, Götterlehre, Leipzig 1989, 56.
} 
und Liminalen auf den Horizont, wird verjagt und ist fehl am Platz. ${ }^{10}$ Phorkyas steht ein für diejenigen, für die niemand einsteht und die in einer Epoche nicht zu ihrem Recht kommen. Entsprechend steht sie den Knechten und Mägden als Schaffnerin vor, stellt die Nichtigen unter das von ihnen verabscheute Zeichen des Nichtigen. Daß ihr Verhältnis zu Helena asymmetrisch ist, daß es nämlich Geschichte nur gibt für das Marginale, als dessen Unterdrücker die Schönheit auftritt, ist die kritische Erkenntnis die Goethe in Helena gewinnt. In dieser Erkenntnis revidiert Goethe sein Lebenswerk.

Während Helena der Gegenstand ist, an dem sich Geschichte erweisen kann, gibt es Geschichte um des Anästhetischen und des Marginalen willen. Phorkyas ist das negative Emblem der Geschichte; sie markiert den Anspruch auf Geschichte. Das Marginale hat in einer jeden Epoche nichts zu hoffen, da es so gut wie nicht existent ist. Daß es dennoch zu Anerkennung kommen kann, verdankt es der Geschichte, denn nur aus der Perspektive einer anderen Epoche mit ihrer veränderten Erscheinungs- und Wahrnehmungsform könnte es gelten. Während Geschichte in Helena zur Katastrophe wird, zum Zusammenbruch des auf ihr Gegründeten, wird sie in Phorkyas zur Hoffnung. Das heißt auch, daß die Geschichte zu einem Ende kommt, wenn es nichts mehr gibt, was zur Erscheinung kommen könnte, wenn nichts mehr am Rand steht, wenn es keine Verlierer mehr gibt. Das Ende der Geschichte zeichnet Goethe dann auch in dem Erscheinen aller am Ende des Helena-Aktes. Dort, am Ende der Geschichte, jenseits der Geschichte der Schönheit, hat Phorkyas das letzte Wort.

Im Folgenden soll die Diskussion mit dem dritten, mittleren Akt des Faust II gesucht werden, ein Akt, der 1827 zunächst selbständig unter dem Titel Helena - Klassisch-romantische Phantasmagorie. Zwischenspiel zu Faust erschienen war und dessen erste Konzeption auf 1800 zurückgeht. In der-notwendig groben-Skizze soll die Struktur der Epochen und Epochenschwellen der drei von Goethe dargestellten Zeitalter Antike, Mittelalter und Neuzeit diskutiert werden. Die These, die ich dabei präsentieren werde, ist eine dreifache. Es soll zum einen gezeigt werden, wie die Epochen jeweils in einer spezifischen Konzeption von "Schönheit" gipfeln, die allen kulturellen Erscheinungen der Epoche Orientierung verleiht wie Jerusalem den Kirchen. Zum zweiten, wie das Ende der Epoche sich nicht nur als

\footnotetext{
${ }^{10}$ Vergl. FA I/7, 694. P176v (Paralipomena).
} 
Zusammensturz an dieser Schönheit erweist, sondern aus ihr selbst hervorgeht. Nicht durch äußere Einwirkung kommen die Epochen zu einem Ende, sondern weil sie in ihrem Inneren auf dem Keim ihrer Überwindung beruhen. ${ }^{11}$ Wie ansonsten nur Hegel und Schelling zeichnet Goethe ein Bild der Geschichte des Abendlandes als einer Geschichte der Erscheinungs- und Zeichenkonzeptionen, und in Überbietung jener sieht Goethe die geschichtliche Dynamik, den Wechsel von Stabilität und Instabilität, in den Erscheinungsweisen selbst begründet. Zum dritten soll gezeigt werden, wie in dieser Geschichtskonzeption das Ende der Geschichte als der Triumph des Marginalen möglich wird.

\section{Antike}

Nach dem trojanischen Krieg flieht Helena, so die Handlung des Stücks, aus der homerischen Antike in Fausts Mittelalter. Ihre Flucht wird stimuliert durch die Befürchtung, Menelas, ihr Gemahl (Menelaos), der sie zurückerobert hat, werde sie, die Kriegs- und Ungücksbringerin, opfern, um vergangene Streitigkeiten um Helena beizulegen und zukünftige zu verhindern. Die Antike ist die Epoche der Heroen, die sich im Streit um den Besitz von Helena befinden. Helena ist dabei nicht der Preis, sondern die Bedingung ihres Heldendaseins. Sie verheißt den Helden den "Ruhm" (8519, 8797), auf Grund dessen sie erst Helden sein können, wie auch sie wesentlich durch ihren zweischneidigen Ruhm geprägt ist: "Bewundert viel und viel gescholten, Helena" (8848). Die antiken Helden ringen entsprechend nicht einfach um eine schöne Frau, sondern um den Besitz dessen, was ihnen Erscheinung, Geltung, Sein und Namen sichert. Schönheit

\footnotetext{
${ }^{11}$ In diesem Punkt komme ich zu einem grundlegend anderen Ergebnis als andere Arbeiten, die das Geschichtsmoment des Helena-Aktes zum Ausgang ihrer Überlegung gewählt haben. So kann die Analyse von Jane K. Brown (Goethe's Faust: The German Tragedy, Ithaca und London 1986) durchaus viele Charakteristika der Epochen bestimmen und analysieren, tut dies aber um den Preis, die Epochen zu rein literaturhistorischen zu verengen. Die Verengung liegt in einer Verharmlosung: Kunst und Literatur seien gewaltfrei, ihre Zerstörung komme von außen ("the world outside the realm of art consists of violence and war," 211). In diesem Export der Gewalt aus der Kunst und aus den Epochen, wird Geschichte, um die es auch Brown geht, unverständlich und unerklärbar. Sie kommt von außen wie die Barbaren, die in Griechenland einfallen. Die radikalere Einsicht Goethes besteht, so meine These, eben darin, daß Kunst und Literatur, aber nicht nur diese, selbst die Gewalt produzieren, der sie zum Opfer fallen, da sie sich nur auf Grund einer solchen Gewalt überhaupt manifestieren können.
} 
ist für Goethes Antike nicht eine bestimmte Wohlgestaltetheit, sondern die Eigenschaft, überhaupt gestaltet zu sein und zu erscheinen. Alle entscheidenden Begebenheiten betreffen dieses höchste Gut der Antike: die Vorgeschichte erzählt den Raub der Helena und trojanischen Krieg, die rückkehrende Helena soll in der Burg zunächst den Schatz ihrer Vorfahren mustern (wobei der Text nahelegt, daß der Schatz als Substitut des Besitzes der Helena fungiert), und schließlich treibt ihre "Opferfurcht" sie zur Flucht, ${ }^{12}$ das heißt, die Furcht, wie ein bloßer Besitz behandelt und fortgegeben zu werden. ${ }^{13}$

Goethe exponiert in den antiken Mythen und Gesängen, die die Helden preisen, den in ihnen verborgenen Index, nämlich die Motivation der Helden, überhaupt besungen zu werden. Nicht um der Tat willen handeln diese Helden, sondern ob der nachträglichen Konstruktion ihrer Tat im Gesang. Ohne Schönheit, ohne Ruhm, wären die Helden anästhetisch, würden dem Vergessen anheimfallen. "Wer keinen Namen sich erwarb, noch Edles will, gehört den Elementen an" (9981-82). Schönheit ist Auszeichnung, sichert den Fortbestand des Namens über den Tod hinaus, mithin ist Schönheitsstreben, Schönheitskampf das Symptom einer Antike, deren Kultur einzig auf der Tartarophobie, der Angst vor ruhmlosem Tod und Vergessen beruht. Der antike Kult der Schönheit, den das achtzehnte Jahrhundert feiert, wird in Goethes Kulturanthropologie zur bloßen Verdrängung der Todesangst. Helena, die Tochter der Lethe, scheidet Sein von Nichtsein, Bestand von Vergessen.

Die Todesverdrängung macht Politik. Erscheinen ist Privileg, und so ruht das Handeln und mit ihm die Politik in den Händen der wenigen, denen die vielen Knechte bloß zur Hand gehen. Die Knechte sind namenlos, ihr Wesen interessiert nicht, es erscheint nicht, hat keine Geltung. "Nicht, was der Knecht sei, fragt der Herr, nur wie er dient" (8794). Sie fallen in die Sphäre des Besitzes. ${ }^{14}$ So ist es bezeichnend, daß eine der wenigen Zeilen der ersten Entwürfe zur Helena, die Goethe unterdrückt hat, eine mögliche Versöhnung von

\footnotetext{
${ }^{12}$ FA I/7, 681. P163 (Paralipomena).

${ }^{13}$ Auch kausale Begründungen, Schmähungen, Wertschätzungen und Gefühle werden regelmäßig in Begriffen des Besitzes ausgedrückt. So gipfelt Phorkyas Schmährede gegen die Mägde der Helena in deren Käuflichkeit: "Naschende Vernichterinnen aufgekeimten Wohlstands ihr! Erobert, marktverkauft, vertauschte Ware du!" (878183). Um so entlarvender ist es, daß der Chor auch Helenas Schönheit als Besitz bezeichnet, sei es auch des "höchsten Gutes Ehrenbesitz" (8517).

14 "Nimm in Besitz den Schatz und sämtlich uns dazu" fordert Phorkyas Helena auf (8806).
} 
Herrn und Knecht, ein ihnen übergeordnetes Recht ausspricht: "Und das heilig[e] Menschenrecht gilt dem Herren wie dem Knecht." In der Antike waltet genau kein Menschenrecht, welches Knechte schützt und Menschenopfer verhindert. Die Privilegierung der Herren über die Knechte drückt sich am deutlichsten in dem Recht zur Veränderung aus:

Das ist des Fürsten Vorrecht daß er alles treu

In seinem Hause, wiederkehrend, finde, noch

An seinem Platze jedes, wie er's dort verließ.

Denn nichts zu ändern hat für sich der Knecht Gewalt. (8556-59)

Der Knecht hütet den Bestand, ohne die Dinge zu verändern. Damit ist der Knecht derjenige, dessen Tun schlicht entfällt, wenn er seine Leistung des Bewahrens vollbracht hat, da er das Produkt nicht beeinflußt. Er soll nur verhindern, daß sich die Zeit an der Ordnung niederschlägt. Seine Funktion besteht darin, nicht zu erscheinen: "Denn schädlicheres begegnet nichts dem Herrscherherrn als treuer Diener heimlich unterschworner Zwist. Das Echo seiner Befehle kehrt alsdann nicht mehr in schnell vollbrachter Tat, wohlstimmig ihm zurück" (8828-31). Entsprechend liegt die einzige Hoffnung des Knechtes nicht in der Bewältigung seiner Aufgaben, nicht in einer Herr-Knecht-Dialektik, sondern darin, daß Veränderung, eine Trübung des Echos, Geschichte, trotz seiner stattfindet. Er kann nicht erscheinen, gelten und sein außer in der Zeit, auf deren Unterdrückung er programmiert ist. Er ist der bloße ethische Anspruch auf Geschichte, der nicht die Gewalt besitzt, diesen Anspruch zu Recht, Gesetz oder Satzung zu erheben.

Goethes Analyse der Antike findet ihren Höhepunkt im Opferkult, dessen Überwindung bereits Iphigenie auf Tauris zur Bedingung der Sozietät erhoben hatte. Nicht irgendwer soll geopfert werden, sondern Helena, so Phorkyas, die sogleich eine Erklärung parat hat: "Unteilbar ist die Schönheit; der sie ganz besaß zerstört sie lieber, fluchend jedem Teilbesitz" (9061-62). In einer früheren Fassung hieß dieser Satz: "Die Schönheit ist ein einzig hohes Gut getheilt nicht denckbar, man zerstört sie lieber selbst zum zehnten Mal/ den Mann veränd[ern] ist nicht gut."16 Der Grund für das Opfer der Schönheit liegt in ihrer Unteilbarkeit, die offenbar so grundlegend ist, daß ihre Teilbarkeit

\footnotetext{
${ }^{15}$ FA I/7, 666. P84 (Paralipomena).
}

${ }^{16}$ FA I/7, 687. H34 (Paralipomena). 
nicht einmal denkbar, oder eben dieses Denken ein Ärgernis ist. Schönheit ist nicht teilbar, auch nicht mitteilbar, sondern das InDividuelle schlechthin. In der Tat kann es ein Heldentum nur geben unter der Bedingung der Individualität der Helden, ihrer Unterscheidbarkeit. ${ }^{17}$ Schönheit besitzen, heißt Individualität haben. Das heißt aber, was die Helden gemeinsam haben oder haben wollen, was sie also teilen, ist das, wodurch sie sich voneinander abgrenzen, ist das Unteilbare, Individualität. Das Dilernma der Individualität besteht darin, daß Individualität nur behauptet werden kann unter der Bedingung ihrer Teilbarkeit.

Da in der Antike nur erscheinen und gelten kann, wer ein Held ist, wer also Individualität besitzt, wird das Erscheinungsgesetz der Antike, Schönheit zu besitzen, eine uneinlösbare Forderung. Die Individualität, die zur Unterscheidung der Helden vorausgesetzt wird und die zur Etablierung eben der vorauszusetzenden Unterscheidbarkeit erlangt werden soll, motiviert nicht nur die desparaten Handlungen der Hellenen, sondern zwingt ihnen auch das Opfer auf.

Was die Helden "zerstör[en]" müssen, ist der "Teilbesitz" des "Unteilbar[en]," das heißt eben dasjenige an der Individualität, was sich ihr entzieht, was sich teilt, was als ein Abstraktum jedem zugänglich ist, nämlich "Individualität." Um der Individualität willen muß die Individualität zu ihrer rituellen Purifizierung zerstört werden. Dies ist der Sinn des Opfers der Helena. Helena, deren Teilbarkeit sich erwiesen hat, muß, um der in ihr verheißenen Unteilbarkeit willen, sterben. Das Opfer der Helena stellt also nicht einfach die Gabe eines hohen Wertes oder des Zankapfels dar, um "die Olympier zu verehren" (8582), sondern ist die Gabe dessen, um dessen willen geopfert wird, der Individualität. In diesem Opfer, das zur Erhaltung der antiken Ordnung notwendig ist, wendet sich die Antike gegen sich selbst. Dabei macht es auch keinen Unterschied, ob Helena tatsächlich geopfert wird oder ob sie vor dem Opfer flieht. Das Zeitalter schlachtet sich selbst.

Eben dies ist die tiefe Zweideutigkeit der Schönheit, die Helena

\footnotetext{
${ }^{17}$ Doch ihre Unterscheidbarkeit wird nicht durch den Namen allein gesichert, denn der Name der Helden ist eigentlich leer. Nur durch den Besitz der Schönheit erlangt der Name die Erhöhung, die den Herrn zum "Heldenherrn" (9137) erhebt. So verneigt sich der Herr vor der Schönheit: "Dem Helden tönt sein Name voran [...] doch beugt sogleich hartnäckigster Mann vor der allbezwingenden Schöne den Sinn" (8520-24), da der Name des Helden nur durch die Schönheit zum Klingen kommt. Die Geltung der Helden, die das ihnen eigenste sein sollte, gründet außerhalb ihrer in der Schönheit.
} 
beklagt-"Denn Ruf und Schicksal bestimmten fürwahr die Unsterblichen zweideutig mir" (8531-32). Sie ist der janusköpfige Stimulus aller Handlungen, der bedroht, was er ermöglicht. So muß sich Helena fragen, als Phorkyas ihr die Zerstörung Trojas anlastet: "War ich das alles? Bin ich's? Werd' ich's künftig sein, das Traum- und Schreckbild jener Städteverwüstenden?” (8840-41). Die Polis beruht auf der Schönheit (so wie Apoll Gott der Schönheit und Städtebegründung ist) und sie findet in Helena ihr Ende. Helena ist das zweischneidige Prinzip, welches die antike Kultur sowohl begründet als auch zerstört, und von deren Zerstörung Helena am Ende selbst erfaßt wird.

\section{Mittelalter}

Das Mittelalter ist für Goethe das Zeitalter der durch Schönheit gestifteten Ordnung, der "schönen Ordnung." ${ }^{18}$ Herrschten die Tyrannen der Antike durch Besitz der Schönheit, so herrscht im Mittelalter die Schönheit selbst über Fürsten und Knechte. Helena setzt sich, gleich nach ihrer Ankunft in Fausts Burg, auf den ihr gewiesenen Thron (9266-72 und 9348-55). Die Schönheitsherrschaft des Mittelalters-wir würden wohl den von Michelet und Burckhardt geprägten Begriff der Renaissance wählen-besteht in einer Ordnungsstruktur, die die Integration aller zum Ziel hat, indem sie jedem seinen Ort zuweist. So ist das Motto dieses Mittelalter das "ein jeder ist an seinem Platz unsterblich" (9552). Ein jeder erlangt seine Geltung durch die Position, die er im Gesamtgefüge einnimmt und jeder ist, was er ist, nur weil er im Verbund der anderen steht. Die Antike dagegen behält das Platzrecht ganz dem Herrn vor, kennt keine ihm höhere Instanz der Ordnung: "Das ist des Fürsten Vorrecht $\mathrm{da} ß$ er alles [...] finde [...] an seinem Platze [...] wie er's dort verließ" (8556-58). Damit nimmt das Mittelalter dem Konflikt von Herr und Knecht die Spitze (und Helenas erste Tat besteht in der Befreiung eines Knechtes, dem in Ketten liegenden Lynceus), da beide ihre Position und Legitimation in einer Ordnung finden.

Diese Ordnung ist die Schönheit, die sich auch in der Architektur durch eine restlose Verplanung des Raumes in rechtwinklige Koordinaten ausdrückt, die keine Penetration durch Fremdkörper, und seien es auch kognitive, duldet: "Dort ist alles senk- und waagerecht

\footnotetext{
${ }^{18}$ So Thomas Zabka in einer informierten Studie, Faust II-Das Klassische und das Romantische, Tübingen 1993, 98.
} 
und regelhaft [...] So starr, so wohl in Fugen, spiegelglatt wie Stahl. Zu klettern hier-ja selbst der Gedanke gleitet ab" (9021-25). Schönheit steckt nicht im Detail, kommt keinem einzelnen Gegenstand zu, sondern liegt eben im Zusammenhang der Dinge begründet, das heißt, in der totalitären Kraft ihrer Verortung. So ist auch in der Rede des Lynceus angezeigt, daß mit dem Auftritt Helenas die Geographie selbst sich verändert: die Sonne "ging auf einmal mir [...] wunderbar im Süden auf" (9224-25).

In der Konstitution des Mittelalters liegt ihre Überlegenheit gegenüber der Antike begründet. Als Menelas mit seinen Truppen versucht, Helena zurückzuerobern, unterliegt er der Stärke von Fausts Heer. Während der Tyrann Menelas 'allein' kämpft, das heißt, nur mit den ihm angehörenden, geltungslosen Untertanen der "kleinen Königsbande" (9456), kann Faust ein größeres Heer um sich sammeln, da er mit vielen anderen verbunden ist. Der mittelalterliche Fürst streitet nicht um Exklusivbesitz der Schönheit, nicht für seinen Namen, sondern für die Ordnung des Ganzen, für das Reich. Keiner von Fausts Mitstreitern muß sein eigenes Sein aufgeben, sondern erhält im Gegenteil als Lohn einen Platz in der Ordnung; konkret umgesetzt wird diese Platzzuweisung, als der siegreiche Faust das eroberte Griechenland unter seinem Heer aufteilt. ${ }^{19}$ Indem der einzelne die Ordnung der Schönheit schützt, dient er sich selbst; Fausts "Befehl vollziehn sie treu, jeder sich selbst zu eignem Nutz" (9496-97). Die Ordnung sichert jedem seinen Ort, den Ort in dem Reich, in dem die einzelnen Fürsten nicht als autonome Tyrannen walten, sondern sich als "Herzöge" zusammentun (9462).

Die Antwort auf die Frage, wie ein solche Gruppe von Fürsten, die nur im eigenen Interesse handeln, überhaupt noch zusammengehalten wird, liegt in Helena. Die Herzöge, eine Vorstudie nennt sie "Lehnsleute," werden zusammengehalten durch das gemeinsame Interesse am Schönen und das heißt durch die bloße Imago des Reiches. Die Imago des Reiches selbst ist der Lohn, der jedem zufällt, der es verehrt, schützt und ihm dient. ${ }^{20}$ Das Reich des Mittelalters ist ein Reich der Bilder und nicht des Besitzes. Und so gibt Lynceus für die

\footnotetext{
19 "Germane du! Corinthus Buchten verteidige mit Wall und Schutz, Achaia dann [...] empfehl ich Gote deinem Trutz. Nach Elis ziehn der Franken Heere, Messene sei der Sachsen Los" und so fort (9466-77). Goethe spielt hier zugleich auf die Völkerwanderung und die kurze Herrschaft der fränkischen Kreuzritter in Griechenland an.

${ }^{20}$ Und so kann jeder der Herzöge die Position einnehmen, die Faust für sich sucht, wenn er Helena anruft: "Bestärke mich als Mitregenten deines grenzunbewußten Reichs, gewinne dir Verehrer, Diener, Wächter all' in Einem!” (9362-64).
} 
Schönheit seinen ganzen Besitz angehäufter Schätze hin, in der Hoffnung nur, daß Helena ihm einen "Blick" gewährt: "O gib mit einem heitern Blick ihm [dem Besitz] seinen ganzen Wert zurück!" (9331-32). ${ }^{21}$ Was von Helena, der Schönheit, erblickt wird, ist Spiegel des Ganzen. Nur in diesen Blicken der Schönheit besteht das Reich. L'état, c'est moi, spricht diese Schönheit in dem Bild, welches die Untertanen von ihr bewahren. ${ }^{22}$

Auf diese bildliche Konstitution des Mittelalters wird einmal bereits in der Antike angespielt, als Phorkyas versucht, Helena das Mittelalter schmackhaft zu machen. Die antike Helena weiß nicht, was "Wappen" sind (9028). Offenbar fehlen die Ikonen politischer Herrschaft in der Antike, da Herrschaft stets auf der unmittelbaren Anwesenheit des Herrn ruht. Das Mittelalter dagegen kennt die Ausweitung des Herrschaftsbereichs durch Bilder (so führte das tatsächliche Mittelalter die Herrscherporträts in den Amtsstuben ein, um auch in deren leibhaftiger Abwesenheit ihre Anwesenheit zu suggerieren). ${ }^{23}$ Mittels der "Bildnerein" und "Wappen" ist das Reich auch dort, wo es nicht ist, ist überall, weil es eben in nichts als solchen Ikonen besteht. Umgekehrt weitet sich der museale Ort durch die "Wappen" tendenziell unbegrenzt aus, umfaßt alle Orte seiner möglichen Geltung. "Dergleichen [Wappen, Bildnerein, Gebilde] hängt in Sälen Reih an Reihe fort, in Sälen, grenzenlosen, wie die Welt so weit" (9042-43).

Man könnte diese Ordnung des Mittelalters als eine symbolische bezeichnen, obgleich das Wort Symbol im Drama selbst nicht fällt, abgesehen allerdings von einem Paralipomenon eben zur MittelalterEpisode des Helena-Aktes, ${ }^{24}$ und obgleich es in der Goethe-Forschung mehr Verwirrung als Klarheit zu stiften scheint. Symbol wäre in diesem Zusammenhang nicht ein Zeichen, welches eine bestimmte Bedeutung trägt, sondern das Durchdrungensein der Elemente von der Ordnung, in der sie figurieren, dergestalt daß im Einzelnen das Ganze versenkt ist wie das Reich im Wappen.

Ein Ende findet dieses Mittelalter nicht in einem Zerfall oder einer Zerstörung ihrer Schönheitsordnung, sondern gerade in ihrer Poten-

\footnotetext{
${ }^{21}$ Auch: "Vor dem Reichtum des Gesichts alles leer und alles nichts" (9355).

${ }^{22}$ Ein durchaus verwandtes Bild des französischen Hofes und den sich in zahlreichen Repräsentationen ausdrückenden Ordnungsstrategien von Louis XIV zeichnet Norbert Elias, Höfische Welt, Darmstadt und Neuwied, 1969.

${ }^{23}$ Vergleiche zur politischen Ikonographie des Mittelalters und den Effigien die grundlegende Studie von Ernst Hartwig Kantorowicz, The King's Two Bodies: A Study in Medieval Political Theology, Princeton 1957.

${ }^{24}$ FA 1/7, 694. P176v (Paralipomena).
} 
zierung. War die Ordnung des Mittelalters hierarchisch in der Schönheit begründet, das Ordnungsgerüst den einzelnen Elementen also äußerlich, so ergeben sich nun Relationen zwischen den einzelnen Ordnungsgliedern unter Ausschaltung des Umwegs über die Herrscherin Schönheit. Statt der Herrschaft und dem Gesetz der Schönheit zu folgen, walten und verbinden sich die einzelnen Elemente nun "eigensinnig" (9542). Nicht ein Mangel, sondern ein Übermaß an Ordnung und Relation führt zur Enthebung der Schönheit aus ihrem Amt, eine Ausdifferenzierung, die das System nicht stützt, sondern unterläuft.

Und so preist der Chor, preist Faust die Ordnung der Natur, in der sich die Wesen verbinden, mischen, aneinanderlehnen. Besondere Betonung erfährt dabei die Präposition "an": "Erhebt sich zweighaft Baum gedrängt an Baum" (9541), "und eigensinnig zackt sich Ast an Ast" (9543) . ${ }^{25}$ Vor allem wird der Liebesbund von Helena und Faust besungen: "Nah und näher sitzen sie schön aneinander gelehnet, Schulter an Schulter, Knie an Knie" (9401-03). Im an vollzieht sich die Veranderung eines jeden, seine Bewegung über sich hinaus. Entsprechend ist der Gott, in dem diese Epoche zu einem Ende kommt, Pan (9538), der Gott des Allganzen, Pan.

Die Relationen und Verästelungen, die sich solcherart zwischen den Dingen ergeben, sind nicht durch eine den Dingen vorgeordnete, mittelalterliche Schönheit programmiert, sondern entstammen ihnen selbst. Deutlich wird diese der totalitären Schönheitsordnung entgleitende Relationalität im Reim, der "Wechselrede" (9376), die der mit dem metrischen Versmaß der Antike vertrauten Helena unbekannt ist. Im Reim geht ein Wort über sich hinaus, verandert. "Ein Ton scheint sich dem andern zu bequemen, und hat ein Wort zum Ohre sich gesellt, ein andres kommt, dem ersten liebzukosen" (9369-71). Aus der Perspektive der Bedeutungen zweier sich reimender Wörter ist der Reim ein Ärgernis, ein möglicherweise Mißverständnisse produzierender, auf jeden Fall aber unnützer Zeitvertreib. ${ }^{26}$ Die sich zwischen den Worten und Dingen bildende Relation verwehrt es, sich einem Nutzen oder einer Bedeutung unterzuordnen. entzieht sich

${ }^{25}$ Alle Hervorhebungen von mir; auch: "Saal an Sälen, Hof an Höfen" (9597).

${ }^{26}$ So Phorkyas: "Buchstabiert in Liebes-fibeln, / Tändelnd grübelt nur am Liebeln, / Müßig liebelt fort im Grübeln, / Doch dazu ist keine Zeit” (9419-22). Phorkyas' Rede, die zunächst -fiebeln, -gr[ie]beln, und -liebeln reimt, unterbricht das unnütze Reimen durch den Hinweis auf die Zeit sowie gleichzeitig durch das reimzerstörende Wort "Zeit." Im Gegensatz zu den Müttern, denen Helena entstammt, pocht Phorkyas auch hier auf die Zermalmung des Bestehenden durch die Zeit, auf die sich kein Reim machen läßt. 
strukturell einer Ordnung wie der mittelalterlichen Schönheit, um stattdessen über jeden Zweck hinauszuschießen. Dieses Inkommensurable nennt der Text Liebe. Sie gab es in der Antike nicht, sie entstammt als Minne dem Mittelalter, führt jedoch zugleich aus dem Mittelalter und der engen Burg hinaus, um eine neue Epoche zu begründen.

"Der Schauplatz verwandelt sich durchaus" (Bühnenanweisung nach 9573).

\section{Romantik ${ }^{27}$}

Die Romantik entdeckt Verhältnisse zwischen den Dingen, die nicht durch externe Gewalt, auch nicht durch die Schönheit gestiftet werden, sondern die aus dem wechselseitigen Bezug zwischen ihnen hervorgehen. Das Zeitalter des neuen Arkadien beruht ganz auf dieser "Liebe"; ihr Prinzip und nicht bloß Produkt ist Euphorion, Helenas und Fausts Sohn. Die Bedingung der Liebe, ihre Voraussetzung, besteht darin, daß es den Dingen eigen sein muß, über sich hinaus gehen zu können und offen zu sein für das, was sie nicht sind. Das Einzelne, gleichviel ob belebt oder unbelebt, muß mehr sein können, als es selbst ist, denn es muß aus seinem Innern "überfließ[en]"(9379), um sich mit dem anderen zu "verweb[en]" (9416). Kein Wesen findet seinen Abschluß in sich selbst. Eben diese Unabgeschlossenheit nennt der Text "Poesie" (9863), verkörpert sie in Euphorion.

In Euphorion gewinnt die Poesie die Oberhand über die bildliche Verfassung des Mittelalters. Findet das Mittelalter in der Imago des Reiches seinen Bestand, so fehlt der nächsten Generation jeder einheitsgebende Rahmen. Poesie ist das Rätsel der Relation, des Über-sich-hinaus-Gehens und des "Übermüt" (9349). Ihren Ort findet die Poesie im "Zwischen," so wie es Euphorions Leitmotiv wird, zwischen den Menschen, Dingen und Wänden hin- und herzuspringen. Poesie besteht wesentlich darin, sich nicht mit einem Wesen zu begnügen und über ein jedes Wesen hinwegzusetzen. Poesie treibt über jedes $\mathrm{Ma}$ hinaus, um ihr einziges $\mathrm{Ma}$ in der Maßlosigkeit, der Verschwendung zu finden. Wie schon der Knabe Lenker sich selbst zugleich als "Poesie" und als "Verschwendung" benannte, so cha-

\footnotetext{
${ }^{27}$ Die abschließende Epoche hat unterschiedliche Benennung erhälten; ich entlehne den Namen "Romantik" dem Titel der Erstveröffentlichung von Helena als "klassischromantische Phantasmagorie."
} 
rakterisiert Euphorion sich als "verschwendrisch" (9846). ${ }^{28}$ Was die Poesie zeigt und bezeichnet, muß sie zugleich überwinden und verschwenden. Nicht im Sagen findet die Poesie ihr Ziel, sondern im Überbieten des Gesagten, nicht im frei sich Anbietenden, sondern nur im "Erzwungenen" (9783, 9795). Die Poesie als Zwang zur Überbietung ist das Wesen des Reims, eines Reims, der sich nicht mit dem Anschluß zweier Worter begnügt, sondern der darauf zielt, über ein jedes Wort hinauszuschießen. Er ist reiner Reim, Beziehung ohne Bezogenes, pure Medialität, absolute Manier.

Aus dieser Poesie der Verschwendung resultiert das Lob des "eigenste[n] Gesang[es]" (9922), der Kult der Selbst-Verwirklichung, für den ein Selbst nicht als gegeben gilt, sondern zur jeweiligen Errungenschaft wird. Der einzelne kann sich nicht mehr mit seinem einfachen Dasein begnügen, sondern kann sich nur in der Überwindung seines einfachen Daseins erweisen. Nicht ein Ort oder Zustand ist Ziel dieser Bewegung, nicht eine Verbindung, wie es in der Liebe noch den Anschein hatte, nicht die Selbst-Findung, sondern die Transgression des Jetzigen, des Selbst und des Nahen in die Richtung eines unbestimmten Fernen. Und so ist Euphorions Parole das "Nur fort! Nun dort" (9874-75), das sich nie mit einem Hier zufrieden geben kann. Die Ablösung vom Boden wird Euphorion wie Antäus zum Verhängnis. Die Poesie des Unbeständigen führt konsequent zur Erschütterung alles Bestehenden, findet ihre Vollendung nur im "Tod" (9884-90). Das politische Programm, welches der poetischen Verfassung Euphorions entspricht, ist die Revolution, deren Leitwort Freiheit heißt und die im Krieg aller gegen alle mündet. Und so ist, gestützt auf eine Bemerkung Goethes an Eckermann, Euphorions Ikarus-Sturz des öfteren als Anspielung auf Byrons fruchtlose Teilnahme am griechischen Unabhängigkeitskrieg gedeutet worden.

Die Kraft der Beziehungsstiftung läßt in ihrer Vollendung keine Beziehung mehr zu, sondern verlangt die permanente Ablösung, die zerstäubende Atomisierung um der Relationalitat willen, die die Auflösung alles zum Wesen Gerundeten bewirkt. In Euphorion schlägt die Begründung der Romantik in ihren Untergang um, "doch du ranntest unaufhaltsam frei ins willenlose Netz" (9923-24).

\footnotetext{
${ }^{28}$ Gegenüber Eckermann hat Goethe auf die Identität von Euphorion und dem Knaben Lenker bestanden: "Der Euphorion ist kein menschliches, sondern nur ein allegorisches Wesen. Es ist in ihm die Poesie personifiziert [...]. Derselbige Geist, der später beliebt, Euphorion zu sein, erscheint jetzt als der Knabe Lenker (Gespräche mit Eckermann, 20.12. 1829).
} 
Diese grobe Übersicht über die von Goethe gezeichnete Geschichte des Abendlands soll hier genügen, um Goethes Thesen weniger zu den einzelnen Epochen, als vielmehr zum Geschichtlichen als solchen aufzunehmen. Es zeigt sich, daß alle drei Epochen strukturelle Ähnlichkeit besitzen nicht in Bezug auf ihre Phänomene, sondern auf ihre Konstitution und Destruktion. Jede Epoche verdichtet sich in einem Wesen, personifiziert in Helena oder Helenas Sohn, das zum Keim ihres Unterganges wird. Nicht in der Abkehr von dem Wesen der Epoche, sondern genau im Gegenteil, in der Radikalisierung, Potenzierung und Verselbständigung eben dieses Wesens findet die Epoche ihr Ende. In allen drei Epochen ist dieser Kern der Epoche eine Formation des Ästhetischen, Epoche mithin je eine Institutionalisierung des Phänomenalen.

Einer anderen Art und Weise, diesen Verhalt auszudrücken, entspräche es, von einer Pathosformel zu sprechen. Jede Epoche verdichtet sich zu einem Wesen, welches einen reaktiven Affekt kultiviert, einen Affekt, der sich als ein Rückkoppelungseffekt dergestalt zu dem Wesen der Epoche verhält, daß er die Epoche als ganzes destabilisiert. Die Privilegierung der Antike schürt Eifersucht, das mittelalterliche Ordnungsgefüge erweckt unkontrollierte Liebe und die freie Relationalität der Romantik erzeugt den Freiheitskult. So gesehen ist der Zerfall einer Kultur, das heißt, das Stattfinden von Geschichte, das Menschliche am Kulturellen.

\section{Das Ende der Geschichte}

Was zu diskutieren bleibt, ist dasjenige, was nach den drei Epochen bleibt, die Topograpie des Endes der Geschichte. Diese findet sich in dem Nachspiel, gleichsam dem Satyrspiel des Aktes. In Euphorions Ikarus-Flug kommt die im Mittelalter ihren Ursprung findende, in der Romantik potenzierte Kultur der Relationalität zu ihrer reinsten Form. Diese ist die Allegorie. ${ }^{29}$ Die Allegorie, die im Exodus oder Schlußgesang ihre Umsetzung findet, begründet keine geschichtliche Epoche mehr, sondern erzwingt das Ende der Epochen und das Ende

\footnotetext{
${ }^{29}$ Explizit macht dies der Knabe Lenker, der sich wie Euphorion als "Poesie" und "Verschwendung" bezeichnet, der sich wiederum selbst als "Allegorie" bestimmt—falls er damit noch ein Selbst bestimmen kann: "Denn wir sind Allegorien" (5531). "Bin die Verschwendung, bin die Poesie. Bin der Poet, der sich vollendet wenn er sein eigenst Gut verschwendet" (5573-75). Zur Kommentierung dieser Rede und zum Status der Allegorie überhaupt vergleiche Werner Hamacher, "Faust, Geld," in Athenäum: Jahrbuch für Romantik 4, (1994) 131-87.
} 
der Geschichte. Geschichte kann es nur geben, solange dem Fluß der Zeit Widerstand geboten wird, und das heißt, solange die Phänomene Halt in einer Schönheit finden. Da die Allegorie aber die Moglichkeit des Andersseins aller Erscheinungen impliziert, fehlt dem Historischen sein Gegenstand, an dessen Widerstand sich Geschichte allein erweisen könnte. Die Allegorie zieht der Geschichte den Boden unter den Füßen weg, indem sie selbst ausführt, was die Geschichte leisten sollte. Neben der Allegorie kann auch keine Schönheit, keine Personifikation des Erscheinens des Erscheinens, bestehen, denn die Allegorie kollabiert das Erscheinen und dessen eigenes Erscheinen. Eine Allegorie zeigt nicht sich selbst, sondern ein anderes; und so besteht sie nur, weil sie stets auch ihr Zeigen zeigt. Daher können Schönheit und Allegorie nicht koexistieren, daher muß Helena die Welt verlassen, als Euphorion in seinem Ikarus-Sprung zu sich selbst kommt. ${ }^{30}$ Die von Heinz Schlaffer in diesem Zusammenhang anhand Hegels Ästhetik entwickelte These, daß die Allegorie ihre systematischhistorische Stellung nicht nur als frühe Kunstform oder Vorkunst, sondern vielmehr als die Form des Endes der Kunst finde, wird von Goethe dahingehend radikalisiert, daß die Allegorie die Form des Endes der Geschichte ist. ${ }^{31}$

Das Ende der Geschichte in der Allegorie bedeutet aber weder das Ende von Erscheinungen, noch das Ende des menschlichen Wirkens.

\footnotetext{
${ }^{30}$ Aus der Unterwelt ruft Euphorion die "Mutter" Helena (9906) zu sich; ihr Abschiedswort an Faust bestätigt ihre Unbeständigkeit, indem sie eben die Beständigkeit eines "alte[n] Wort[es]" Calderons erweist: "Ein altes Wort bewährt sich leider auch an mir: Daß Glück und Schönheit dauerhaft sich nicht vereint. Zerrissen ist des Lebens wie der Liebe Band" (9939-41).

${ }^{31}$ Schlaffers grundlegende Arbeit hat eine Rehabilitation der Allegorie im Denken des späten Goethes geleistet, die inzwischen ihr berechtigtes Echo in der Forschung nicht nur zu Goethe gefunden hat (Faust Zweiter Teil. Die Allegorie des 19. Jahrhunderts, Stuttgart 1981). Es sei daher erlaubt, hier nur auf eine problematische Seite von Schlaffers Argument hinzuweisen, problematisch in Bezug auf die Reichweite der Allegorie in Faust II. Schlaffer hält an der Allegoriekonzeption der Aufklärer des achtzehnten Jahrhunderts fest, die Allegorien als tote, gespensterhafte Zeichen betrachtet. Doch die Allegorien des Faust II lösen sich ab von der Vorstellung einer verkörperten Abstraktion (z. B. "der Winter"), um stattdessen die mögliche Zeichenhaftigkeit sowohl des Darstellenden und des Dargestellten zu betonen, dergestalt daß kein Allgemeinbegriff die Allegorie je mit einer abschließenden Deutung sättigen könnte. Selbst wenn man mit Schlaffer übereinstimmt, Helena sei eine Allegorie des aufklärerischen Typus (obgleich ihr 'Gemeintes' sich erst aus der Epoche, dem Kontext, ergibt und sich damit als wandelbar erweist), zeigen die Allegorien des Exodus keine abstrakten Allgemeinbegriffe mehr, sondern zeigen 'etwas als etwas', um in keinem 'etwas' als einem Gemeinten ihren Abschluß zu finden. An diesen Lebensgeistern des Exodus erweist sich Schlaffers Diktum "der allegorische Charakter einer Figur nimmt in dem Maße zu, wie sie an Leben verliert" (117) als durchaus falsch.
} 
Entgegen dem Allegorieverständnis der Aufklärung sind die Allegorien des Exodus keine toten, sondern sehr lebensfrohe Geister. Was übrig bleibt, wenn die Kultur zu Ende geht, ist, dem alten Schema zufolge, die "Natur," sind die unverbundenen "Elemente"(9982), der Chor, "wir im Hintergrund" (9974), die Namenlosen (9981). Die vollständige Emanzipation des Marginalen steht bevor. Die Randwesen verweigern es, Helena, Euphorion und Panthalis in die Unterwelt zu folgen, wo sie weiterhin ihr Schattendasein fristen würden ("fledermausgleich zu piepsen, Gefluster, unerfreulich, gespenstig" [9979 80]). ${ }^{32}$ Nun erst können sie zu ihrem Recht kommen in einer Zeit, die nicht durch Rechte und nicht durch die Restriktion einer Kultur begründet ist. Sie hatten in der Geschichte auf nichts zu hoffen als auf das Ende der Geschichte. Dieses Ende ist nun erreicht, nun dürfen diese Gespenster die Szene jenseits des Historischen füllen, dürfen zu den Protagonisten einer Zeit ohne Protagonisten aufrücken. Nur am Ende der Geschichte können die Verlierer des Geschichtlichen erscheinen. Ihre Erscheinung mündet nicht in der Errichtung einer Demokratie, nicht einmal einer Phänokratie, sondern nur der Abschaffung aller Herrschaft in der Anarchie, deren historisches Vorbild Goethe in der Revolution sieht. In dieser Post-Historie erhält nicht ein Subjekt vor dem anderen das Wort, sondern nun sprechen, so heißt es, "alle" (9985). "Alle" werden nicht einfach im Text benannt und angesprochen, sondern werden die dramatis figurae, werden zur Summe aller möglichen und auch unmöglichen dramatis figurae. Das emphatische "alle" kann an dieser Stelle nicht auf ein Tutti des Chors reduziert werden, der zuvor sprach und der sich noch nicht in vier Teile aufgeteilt hat. Niemand spricht nun nicht mehr. "Alle" befestigen in ihrem ersten Wort sogleich ihren Status als diejenigen, denen es unumkehrbar zukommt zu erscheinen, unumkehrbar auch durch Geschichte: "ALLE. Zurückgegeben sind wir dem Tageslicht, [...] zum Hades kehren wir nimmer" (9985-98). Geschichte kann es nun auch schon deshalb nicht mehr geben, weil es ohne das Sinn- und Lichtzentrum Helena auch keinen Rand mehr gibt, der auf sein Recht pochen könnte. Alle sind schon da.

Die Form, die das Erscheinen aller nimmt, die einzige Form des Erscheinens, in der ein Wesen nicht auf Kosten des anderen erscheint, ist die Allegorie. Etwas erscheint nicht auf Kosten eines anderen, gerade weil es als ein anderes und in einem anderen erscheint, und

\footnotetext{
${ }^{32}$ Diese sonderbare Rede erinnert an die Verwandlung der webenden Töchter des Minyas in Fledermäuse, nachdem diese es verweigert hatten, Bacchus zu huldigen. Dieses wird der Chor nicht versäumen (Ovid, Metamorphosen, IV, Vers 389-414).
} 
insofern strukturell unfähig ist, irgendetwas auszuschließen. Die namenlosen Chortiden werfen ihr menschliches Sein ab, um als "Geister" in die Natur einzugehen:

Ewig lebendige Natur

Macht auf uns Geister,

Wir auf sie vollgültigen Anspruch. (9989-91)

Vollgültig ist der wechselseitige Anspruch, weil ihm nichts entgegengestellt werden kann, denn ohne die Schönheit gibt es keinen Halt, keinen Einhalt, der die Vermischung des Natürlich-Realen und des Geisterhaft-Imaginären verwehren könnte, da eben die Trennung beider nur innerhalb einer Kultur der Wesen möglich ist. Die wechselseitige Beanspruchung von Natur und Geistern führt zur Saturierung der Zeichen mit Leben und der Bedeutungsanreicherung im Lebendigen. In dieser Nach-Zeit, der Moderne, endet nicht das Erscheinen, sondern wird im Gegenteil übermächtig, da nur die epochale Gewalt der Schönheit die Erscheinungen beschränken, regulieren und je privilegieren könnte. Ein jedes ist nun potentiell die Hülle eines anderen. Und so bleibt von Helena nichts übrig als der Schleier, in den sich ein jeder hüllen könnte, und von Euphorion das Instrument und "Kleid," das einem jeden zur Staffage als Dichter genügen könnte, wie Phorkyas sogleich erkennt (9955-61). ${ }^{33}$

Diese letzte Szene erlangt als Inszenierung der Zeichenwerdung der Welt, als Allegorese, Lesbarkeit. Ihr Horizont ist die Schrift. Der Chor, der seine neue Heimat in den "Elementen" (9982) findet, wird, dem Griechischen gemäß, zum Alphabet. Der Schlußgesang präsentiert die Evolution der Schrift von der Handschrift zur Druckschrift und vom solitär arbeitenden Dichter zum wilden Lesebetrieb der neuen Öffentlichkeit. Während die geschichtliche Zeit in der Schönheit kulminiert, so die Post-Historie in der Schrift. Doch während in den geschichtlichen Epochen die Schönheit selbst als ihr Kern hervortrat, tritt die Schrift nie unmittelbar auf, sondern zeigt sich nur in Anspielungen, Verweisen und als Allegorie. Wenn der Exodus die Zeichenwerdung der Welt inszeniert, dann beschreibt er diesen Vorgang nicht, sondern stellt ihn seinerseits allegorisch dar, denn er benennt seinen Gegenstand an keiner Stelle. Der Exodus ist-falls es in einem

\footnotetext{
${ }^{33} \mathrm{Da}$ der Schleier bei Goethe alles andere als belanglos, sondern entscheidend ist in der Konstitution von Subjektivität, hat Rainer Nägele betont und zum Anlaß einer Studie zur Figur des Voyeurs gewählt, Reading after Freud: Essays on Goethe, Hölderlin, Habermas, Nietzsche, Brecht, Celan, and Freud, New York 1987, 23-45.
} 
Zeitalter ohne Bestand noch ein solches Sein geben kann-eine Allegorie der Allogerese. Indem diese Allegorie der Allegorie der Schrift gilt, zeigt sie, daß die Allegorie es sogar vermag, sich selbst zu zeigen, sich selbst als Allegorie, als Zeichenwerdung. Das heißt aber, sie zeigt sich, und sie zeigt sich nur als sie selbst. Phorkyas legt nun die Maske ab, zeigt sich "als Mephistopheles" (Bühnenanweisung nach 10038). Oder zeigt sie sich nur "als Mephistopheles"? ? $^{34}$ In der Ununterscheidbarkeit von dem "sich selbst" und "als sie selbst" sprießt und rankt die Allegorie.

Entsprechend kann auch nicht die Richtigkeit meiner Deutung (der Apotheose der Schrift) behauptet werden, sondern nur die Unabweislichkeit ihrer Möglichkeit, obgleich sie bisher von keinem anderen Leser der Helena vertreten worden ist. Der erste Teil des Chores beschreibt die deutsche Schrift mit ihren "Flatterhaaren," einem terminus technicus der Kalligraphie (9992-98). Der zweite Teil des Chors findet sich ein, wo die Felswände ein Echo werfen, und deutet das Bild des Echos um zur Vervielfältigung durch die Druckerpresse als "erschütternde[s] Verdoppeln, dreifach, zehnfach hintennach" (999910004). Der dritte Teil zieht mit den Flüssen in die Täler und zeigt die Ausweitung des durch Schrift und Zeichen Erfaßten, das "Bezeichnen" (10009), Zeichenwerden, der Landschaft (10005-10). Der vierte Teil des Chors schließlich zeigt die Produktion und Rezeption der Literatur in der konventionellen Allegorie der Weinlese (10011-38).

Der Dichter-Winzer kultiviert den Buchstaben ("wo am Stab die Rebe grünt," Stab der Name des vertikalen Balken der Druckschrift), der über die Zwischenstation der Weinkeller-Archive schließlich im orgiastischen Fest der Literati des Dionysos mit ihren frakturartig "gespaltne[n] Klauen" zerstampft und konsumiert wird. Nicht die Trauben sind das Ziel, sondern deren Transformation und Deformation in der Weinherstellung. Nicht in der Bewahrung des Gesagten, sondern gerade in der Transsubstantialisierung und der Erzeugung phantastischer und wilder Bilder in der Trunkenheit wird die Weinlese zum Sinnbild der modernen Zeichenwelt.

Tatsächlich ist der Akt von Referenzen auf die Mythen der Schrifterfindung durchzogen. So erscheinen die drei am meisten genannten Erfinder der Schrift, die die Antike kennt, Hermes, Dionysos und Silen, im Akt stets in einem Kontext, der den Bezug zur

\footnotetext{
${ }^{34}$ Das Extraordinäre der Regieanweisungen am Ende des Aktes, die keine Vergleichsstelle im Faust findet, betont Hans Arens, Kommentar zu Goethes "Faust II," Heidelberg 1989, 737.
} 
Schrift nahelegt ${ }^{35}$ Eine Anspielung auf die Schrift gibt auch Phorkyas in der rätselhaften Rede, in der sie die Chortiden mit Kranichen vergleicht. Aus dem Flug der Kraniche habe, so heißt es bei Creuzer, Hermes die Anregung für die Erfindung der Schrift erhalten..$^{36}$ Auch die Vision des Chores von Euphorions Tod beschwört die Schrift:

\author{
Magst nicht in Burg und Wald \\ Friedlich verweilen, \\ Suchen wir alsobald \\ Reben in Zeilen, \\ Reben am Hügelrand; \\ Feigen und Apfelgold. \\ Ach in dem holden Land \\ Bleibe du hold! (9827-34)
}

Die Trauerarbeit zum Tode Euphorions manifestiert sich in dem Suchen von "Reben in Zeilen" in denen die Poesie offenbar fortlebt. Der Wein ist nun aber nicht mehr frei zu haben, erklingt nicht mehr im Gesang der "Oper," sondern muß den "Zeilen" abgerungen werden, in der Arbeit von Zeile zu Zeile durch einen ganzen Text von Zeilen. Diese Zeilen sind nicht nah, nicht greifbar, sondern stehen am "Hügelrand," so daß sich an der Horizontlinie ihr Silhouettenbild abzeichnet, ein Bild welches durchaus an ein Schriftbild erinnert, eine Schrift, die wie Phorkyas ihren Ort stets nur am "[R]and" hat.

In dem Chorlied erklingt zugleich ein Echo auf die Erkundschaftung des gelobten Landes durch das aus Ägypten kommende Volk Israel (Moses 4; 13, 24). Dort heißt es, die ausgesandten Kundschafter kehrten beladen mit üppigen Reben, Feigen und Granatäpfeln zurück, um allerdings sogleich von der Unzugänglichkeit des gelobten Landes zu künden, das durch schreckliche Riesen bewohnt sei. Die Kunde der Boten stellt das von ihnen Vorgeführte, die Früchte, zugleich in die unerreichbare Ferne, behauptet, die paradiesischen

\footnotetext{
${ }^{35}$ Dionysos und Silen erscheinen im Exodus in der Weinlese, Hermes dagegen als derjenige, dessen "Stab" (9117) Helena und den Chor aus der Antike ins Mittelalter leitet und dessen diebische Jugendtaten zum Modell des Euphorionkindes werden.

36 "Euch find' ich nun, ihr Frechen, aus der Fremde her mit Übermut ergossen, gleich der Kraniche laut-heiser klingendem Zug, der über unser Haupt, in langer Wolke, krächzend sein Getön herablschickt, das den stiller Wandrer über sich hinauf/ zu blicken lockt" (8764-69). Die Versuchung für den Wanderer liegt offensichtlich in dem bloßen Blick in die Ferne, in der Erfindung vielleicht der alles entfremdenden Schrift. Vergl. (Georg) Friedrich Creuzer, Symbolik und Mythologie der alten Völker, besonders der Griechen, 2. Aufl. Leipzig und Darmstadt 1819, reprograph. Nachdruck, New York 1978, Band 1, 366. Neben Hermes als Schrifterfinder wird dort auch Palamedes genannt.
} 
Früchte verhießen kein Paradies. Die Schrift hat eben diese Position eines double bind inne, ist Zeichen dessen, was sie vorenthält. Der Buchschmuck der Bibelausgaben des siebzehnten und achtzehnten Jahrhunderts hat regelmäßig eben diese Episode umgesetzt durch das Initial des Buchstabens $\mathrm{H}$ oder $\mathrm{N}$ in einer Darstellung zweier Kundschafter mit den von einem Stab herabhängenden Früchten, Frucht und Schrift ineinanderschwenkend.

Um es noch einmal zu sagen: nicht ob diese Lektüre richtig ist, steht auf dem Spiel, sondern die Schwierigkeit, vielleicht Unmöglichkeit, sie abzuweisen. Der Ungewißheit der "Bedeutungen" begegnet die Post-Historie durch ein Anwachsen der Verifizierungsmechanismen und der hermeneutischen Verfahren, die es sich zur Aufgabe machen, Anspielungen und Chiffrierungen zu entziffern. Doch die Institutionalisierung der akademischen Disziplin der Hermes-Kunst Hermeneutik zu Goethes Lebzeiten ist nur das Symptom einer Krise, nicht ihre Aufhebung. Im Gegenteil: die Hermeneutik besteht auf Grund der Uneinlösbarkeit des von ihr Geforderten, besteht in der Ausweitung und Forderung der Krise des Verstehens.

In der Einführung des Hermetischen, der Schrift, gibt Goethe einen Hinweis darauf, was nach der Geschichte, nach der Schönheit kommt. Dies geschieht im Proszenium. Es heißt nach dem letzten gesprochenen Wort des Helena-Akts, im letzten gedruckten Wort des Aktes, daß sich Phorkyas riesenhaft aufrichtet, um das Stück zu "kommentieren." Was sie aber sagt, legt Goethe nicht mehr $\mathrm{au}(\mathrm{k})$ toritär fest. Nach der Geschichte der Schönheit gibt es noch Kommentierung. Und mit der Kommentierung könnte und müßte es auch eine Pluralität der Kommentierungen geben. Hegels These vom Ende der Geschichte wird von Goethe als Beginn des Streites der Auslegeverfahren fortgeschrieben. Den Platz der Geschichte nimmt in der Post-Historie die Hermeneutik ein. Diesen Streit der Kommentierungen und Interpretationen sieht Goethe um 1800 eingeläutet,—eine in der Tat bemerkenswerte Voraussicht. Mit diesem Streit der Auslegung wäre vielleicht auch eine Geschichte der Deutungen zu denken, eine Geschichte freilich, die Epochen nicht auf Grund ihres inneren Wesens bildet, die überhaupt keine Epochen bildet, sondern eine Geschichte der Unterbrechungen und Stillstellungen nach Vorbild des ständig einschreitenden Teufels. Die Geschichte der Schrift wäre eine Geschichte der Unterbrechungen.

Indiana University, Bloomington 